\title{
Correlating Navigation Barriers on Web 2.0 with Accessibility Guidelines
}

\author{
Letícia Seixas Pereira ${ }^{(\bowtie)}$ and Dominique Archambault \\ Université Paris 8 Vincennes-Saint-Denis, \\ 2 Rue de la Liberté, 93526 Saint-Denis, France \\ leticia.seixas-pereira02@univ-paris8.fr
}

\begin{abstract}
The constant emergence of new resources and interaction possibilities brought by web 2.0 brings a constant urge of keeping in track how those new elements can affect the interaction of people with disabilities and how far current re-search has managed to address existing problems. This paper presents the results of an empirical study designed to gather navigation barriers affecting blind people when interacting with some widely used web widgets - such as Popup windows, Auto Suggest Lists, etc. The aim is to correlate those barriers with WCAG 2.0 and ARIA guidelines. From this point of view, it is possible to determine which barriers are being covered by current guide-lines and which ones are still on the wild. As conducting users' evaluations are really costly, the more barriers can be eliminated beforehand, the more useful those sessions can be.
\end{abstract}

Keywords: Web accessibility $\cdot$ User evaluation $\cdot$ Accessibility guidelines Navigation barriers $\cdot$ Web 2.0

\section{Introduction}

Once characterized by static pages and a passive environment, the web is currently a dynamic structure, that, through the use of several components (widgets), it combines a desktop interface and functionalities with the wide range of a web application $[6,10]$. However, concerning the accessibility of these pages, all this interactivity represents a problem for users with disabilities, since assistive technologies are still trying to cope with this interaction model and dynamic updates [5, 6]. Furthermore, in order to make an accessible web 2.0 it is essential to comprehend how users interact with these dynamic contents and highly interactive components [6]. With that in mind, we correlated WCAG guidelines and WAI-ARIA recommendations that can be applied on the development of accessible web widgets. Following that, an empirical study was conducted with eleven blind users to gather a collection of barriers faced by them when interacting with dynamic components. The goal of this research was to link both specifications and analyze how far they can assure an accessible dynamic environment and which problems are still not covered by them. 


\section{Background}

Power et al. [13] and Vigo and Harper [15], concentrate the efforts on the perspective of understanding user behavior and navigation strategies, however, with different goals. While the first one aims towards personalized web applications for people with disabilities, the second one aims towards navigation problems in real-time. Moreover, about code standardization, one significant attempt to create accessible dynamic content is WAI-ARIA. Published in 2014 by the W3C, it adds roles, states and properties to HTML tags to identify user features and their connection and states. It also offers the resource of communicating some updates to the screen reader [16]. Regarding methods for evaluating the accessibility on dynamic updates, Abu Doush et al. [1] and Fernandes et al. [9], proposed the evaluation of these pages by triggering all JavaScript events, i.e. generating all possible code changes, in order to check code compliance with WAIARIA and WCAG 2.0 specifications respectively. However, one considerable problem still remains: although it is essential to enable users to access the information, it is also extremely important being sure that they are able to make efficient use of it [4], i.e. that they understand interact with the content. Yet, this subject is not yet being sufficiently addressed by current researches. Given that, we aim to connect all those points, by finding out existing gaps between currently guidelines and navigation barriers, through gathering interaction problems from an empirical study and correlating them with WCAG 2.0 guidelines and WAI-ARIA Authoring Practices 1.1.

\section{Method}

We conducted an exploratory study case with eleven blind users who undertook several tasks on a variety of pre-defined websites, in order to observe their interaction and gather a collection of problems encountered by them while performing online activities.

\subsection{Website Selection}

Websites to be used was first based on a list provided by Alexa ${ }^{1}$ web analytics tool, with the most accessed ones in France and, to ensure a dynamic sample, the sites at the top of the list were analyzed from the tell-signs described by [7]. Those who had the highest number of web widget were selected. Next, we elaborated tasks for each website based on its main activities as well its own purpose and also considering an activity that required an interaction with the widgets available on their pages.

\footnotetext{
${ }^{1}$ http://www.alexa.com.
} 


\subsection{Participants}

This study was conducted with eleven blind users, between 36 and 66 years old. Most of them self-declared as experienced users and working with computer related activities, while the other two participants declared having an average familiarity with computers and internet. One participant used Voice Over and the rest of them used JAWS as their main screen reader software. Some of them used NVDA as a second screen reader during the evaluations, changing between tasks. Five of them counted with a braille display to assist their navigation. To exclude some external factors, all evaluations were conducted in their familiar environment (home/work places) and their usual assistive technologies, computers and software configurations were used.

\subsection{Design}

Evaluations began with us informing the participants about the study goal and how it would be conducted. Next, we ask them to sign a consent form and applied a profile form. The participants were encouraged to report any problems, using the "think aloud" verbal protocol [8], and after finishing the tasks from a website, they were asked to summarize their difficulties and how they affected task execution. Each evaluation was registered by an external digital camcorder and additional notes.

\section{Data Analysis}

In the first place, all data collected (notes, videos, forms and user reports) from participants was reviewed to identify barriers during their interaction with selected websites and their respectively widgets. Each barrier was labeled and categorized by the following items: (1) widget: corresponding element; (2) severity (minor/significant/ critical): level of disruption caused by the barrier (from a minor shift in the interaction to an interruption of current task); (3) signalized/identified: whether the barrier has been signalized by the user or identified by the researcher; (4) impact: description of the consequences of the barrier in the interaction and task execution; (5) description: description of the barrier itself. Further, a second round of categorization was realized to highlight the instances of same barriers. For this, each instance was classified by its frequency, assistive technology used, user experience (from 1: none to 5: advanced), severity and general description. The final step consisted in correlate each final barrier with WCAG 2.0 guidelines and WAI-ARIA authoring practices to determine which of these barriers could be avoided by using recommendations and to identify the gap between development specifications and interaction barriers. 
Table 2. Final Barrier 1: lack of feedback from a PopUp Content.

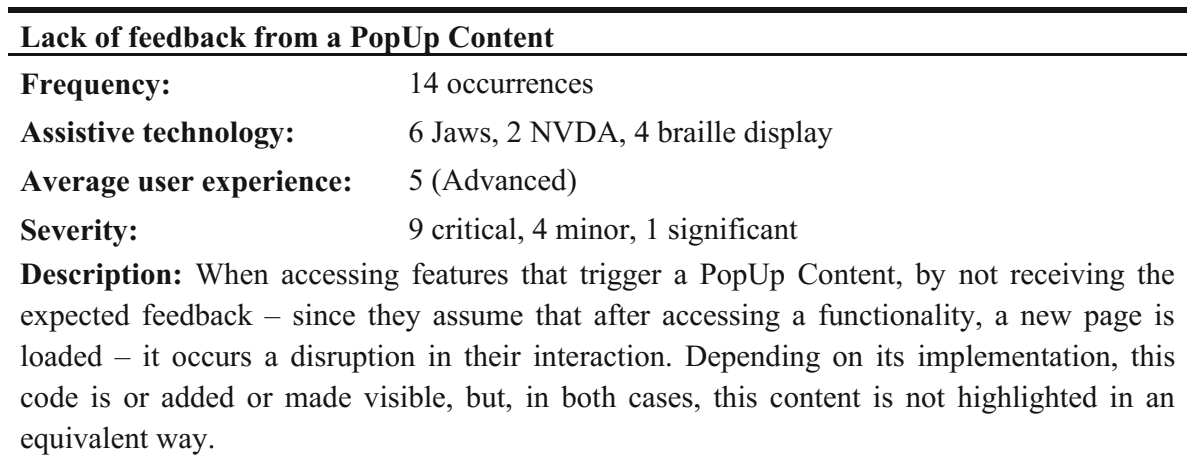

\section{WCAG 2.0 related guidelines:}

- Guideline 1.3: Success Criteria 1.3.1, 1.3.3: Content created should be presented in different ways without losing information or structure. Also, this structure must be reflected in the code, i.e. programmatically determined, and should not rely on sensory characteristics. But, when a PopUp is triggered and opened, visually, it is possible to recognize the highlight of the information, that, without the proper screen reader notification, it is not perceived.

- Guideline 2.4: Success Criterion 2.4.3: In a navigation sequence, components should receive focus in an order that preserves meaning, helping users locate themselves. Keyboard focus was not located inside the modal box, i.e. inside the functionality, and the primary window that should be blocked was navigable by the keyboard.

- Guideline 3.2: Success Criterion 3.2.4: Pages should appear and operate in predictable ways and, components that have the same functionality within a set of web pages should be identified consistently. But, while some menu options linked to another page, other triggered a PopUp Content, making their behavior completely unpredictable by the user.

- Guideline 4.1: Success Criterion 4.1.2: It assists a maximum compatibility with assistive technologies programmatically determined by the use of states, properties and their corresponding values. By using HTML tags name, role and value, assistive technologies could provide notifications on changes in those pages, avoiding the lack of feedback.

\section{WAI-ARIA 1.1 related Authoring Practices 1.1:}

- 3.8 Dialog (Modal): < dialog >: Indicates that the element is a descendent window. 
Table 3. Final Barrier 2: difficulty in the perception of contents and sections in a Carousel.

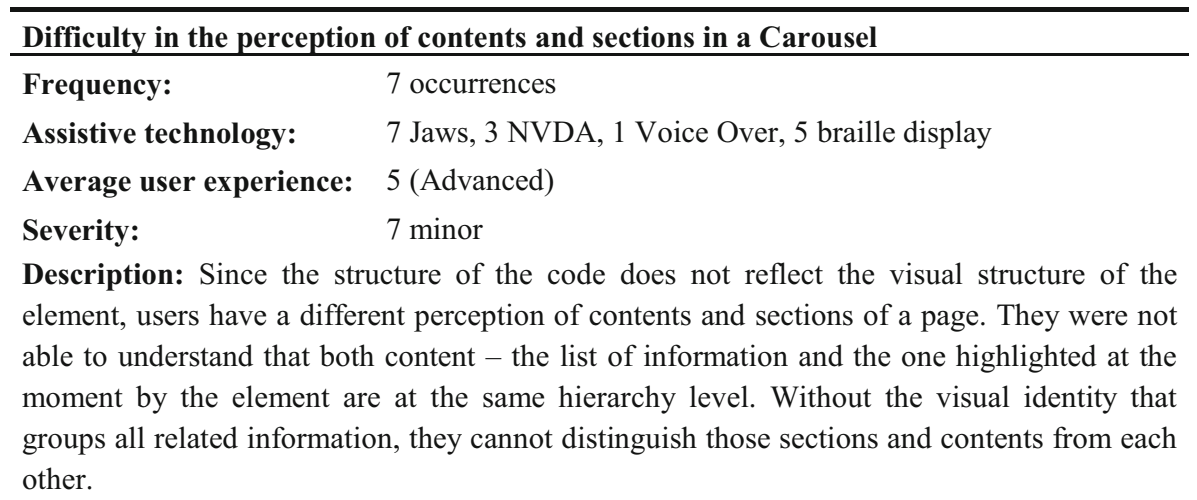

\section{WCAG 2.0 related guidelines:}

- Guideline 1.3: Success Criteria 1.3.1, 1.3.2, 1.3.3: Content should be presented in different ways without losing information or structure and it should maintain its structure and relationships presented in the same way, containing the same meaningful sequence and also it should not rely on sensory characteristics for being understood and operated. But, a content available by a Carousel when presented by a screen reader, did not reflected the same hierarchy and grouping that the visual one, changing its meaning and its relevance.

- Guideline 2.2: Success Criteria 2.2.3: As the Carousel is a based time widget, it should provide users enough time to read and use its content. Also, timing should not be an essential part of the activity, however, as the highlighted information changed, it also changed the hierarchy of main headings, altering the general page structure.

- Guideline 2.4: Success Criteria 2.4.10: Section headings should be used to organize the content and to help users navigate, find content and determine where they are. But, by changing the highlighted information, Carousel also changed headings structure, leaving the user extremely disoriented.

WAI-ARIA 1.1 related Authoring Practices 1.1: No recommendation was found.

- Carousel: (1) Elements at the same hierarchy level should be equally identified in the code, respecting their corresponding section grouping; (2) Page main structure and information should not be altered without a user action or a notification; (3) If what is changed is only visual, i.e., one content at a time being visually highlighted from a list, it should be kept along with control layout and presentation code (CSS);

- Tabs: (1) Titles of tabs and their corresponding contents must have the same logical structure as that displayed visually when read by the screen reader; (2) Users must be able to recognize the titles of the element and also manipulate them only with the use of the keyboard; (3) Visual hierarchical structure of the tabs must be reflected in the code so that the software is able to accurately reproduce the available content. 
Table 4. Final Barrier 4: inappropriate identification of the element "Tabs".

\begin{abstract}
Inappropriate identification of the element "Tabs"
Frequency:

9 occurrences

Assistive technology: 5 Jaws, 1 NVDA, 2 braille display

Average user experience: 5 (Advanced)

Severity:

5 critical, 2 significant, 2 minor

Description: Since the code structure does not reflect the visual, if the identification is not adequate, the user does not identify the logic - title, link, list of information - in the content. In such cases, or he does not know how to interact with the element, not having access to the information at all, or he accesses information that does not correspond to the expected one.

WCAG 2.0 related guidelines:

- Guideline 1.3: Success Criteria 1.3.1, 1.3.2, 1.3.3: Content should maintain its structure and relationships presented in the same way, containing the same meaningful sequence, also it should not rely on sensory characteristics for being understood and operated. But, the content provided, when presented by the screen reader, is not equivalent to the structure visually displayed, representing different relationships and navigation sequences.

- Guideline 2.4: Success Criteria 2.4.3, 2.4.6, 2.4.10: Users must be able to locate themselves and section headings and focus order should reflect the same structure as the visual one.
\end{abstract}

WAI-ARIA 1.1 related Authoring Practices 1.1:

- 3.2.1 Tabs: $<$ tablist $>$ : container for a set of tabs; $<\mathrm{tab}>$ : tab control; $<$ tabpanel $>$ : tab container.

\title{
6 Conclusions
}

As already shown in several researches [2, 3, 11, 12, 14, 17], relying only in accessibility guidelines is not enough to ensure an accessible website, especially if taking into account the growth on web 2.0 related technologies. Automatic evaluation tools are capable to identify a great number of possible barriers, however, some of them can only be perceived through real user interaction. From the conducted study case we analyzed how currently guidelines such as WCAG 2.0 and WAI-ARIA combined can and should be used to promote web accessibility for dynamic pages. We also perceived that there is still a gap in accessibility guidelines, since it depends on the interpretation of the relationships between the elements present in the page. For that, it becomes necessary more studies on how users interact with these widgets to check which barriers are still not covered by current guidelines in order to direct our efforts.

As this is an ongoing research, future studies include users with different kinds of disabilities and also the development of a tool for assisting dynamic pages evaluations based on navigation barriers, evolving these conclusions into code checking items. 
Acknowledgments. This work was supported by Coordenação de Aperfeiçoamento de Pessoal de Nível Superior (CAPES), Brazil, grant Doutorado Pleno no Exterior.

\section{References}

1. Abu Doush, I., Alkhateeb, F., Al Maghayreh, E., Al-Betar, M.A.: The design of RIA accessibility evaluation tool. Adv. Eng. Softw. 57, 1-7 (2013)

2. Brajnik, G.: Validity and reliability of web accessibility guidelines. In: Proceedings of the 11th International ACM SIGACCESS Conference on Computers and Accessibility ISSU, pp. 131-138 (2009)

3. Brajnik, G., Yesilada, Y., Harper, S.: Testability and validity of WCAG 2.0. In: Proceedings of the 12th International ACM SIGACCESS Conference on Computers and Accessibility ASSETS 2010, p. 43. ACM Press (2010)

4. Brown, A., Jay, C., Harper, S.: Audio presentation of auto-suggest lists. In: Proceedings of the 2009 International Cross-Disciplinary Conference on Web Accessibililty (W4A), pp. 5861 (2009)

5. Brown, A., Jay, C., Harper, S.: Tailored presentation of dynamic web content for audio browsers. Int. J. Hum. Comput. Stud. 70(3), 179-196 (2012)

6. Chen, A.Q.: Widget identification and modification for web 2.0 access technologies (WIMWAT). ACM SIGACCESS Access. Comput. 96, 11-18 (2010)

7. Chen, A.Q., Harper, S., Lunn, D., Brown, A.: Widget identification: a high-level approach to accessibility. World Wide Web 16(1), 73-89 (2013)

8. Ericsson, K.A., Simon, H.A.: Protocol Analysis: Verbal Reports as Data. Revised edition (1993)

9. Fernandes, N., Costa, D., Neves, S., Duarte, C., Carriço, L.: Evaluating the accessibility of rich internet applications. In: Proceedings of the International Cross-Disciplinary Conference on Web Accessibility - W4A 2012, p. 13. ACM Press (2012)

10. Fortes, R.P.M., Antonelli, H.L.: Avaliação de Acessibilidade e Usabilidade em RIA. In: Anais do XXII Simpósio Brasileiro de Sistemas Multimídia e Web, vol. 3, Minicursos, Teresina, Piauí, 8 a 11 de novembro 2016 (2016)

11. Leuthold, S., Bargas-Avila, J.A., Opwis, K.: Beyond web content accessibility guidelines: design of enhanced text user interfaces for blind internet users. Int. J. Hum. Comput. Stud. 66(4), 257-270 (2008)

12. Power, C., Freire, A., Petrie, H., Swallow, D.: Guidelines are only half of the story: accessibility problems encountered by blind users on the web. In: Proceedings of the 2012 ACM Annual Conference on Human Factors in Computing Systems - CHI 2012, p. 433. ACM Press (2012)

13. Power, C., Petrie, H., Swallow, D., Murphy, E., Gallagher, B., Velasco, C.A.: Navigating, discovering and exploring the web: strategies used by people with print disabilities on interactive websites. In: Kotzé, P., Marsden, G., Lindgaard, G., Wesson, J., Winckler, M. (eds.) INTERACT 2013. LNCS, vol. 8117, pp. 667-684. Springer, Heidelberg (2013). https://doi.org/10.1007/978-3-642-40483-2_47

14. Rømen, D., Svanæs, D.: Validating WCAG versions 1.0 and 2.0 through usability testing with disabled users. Univers. Access Inf. Soc. 11(4), 375-385 (2012) 
15. Vigo, M., Harper, S.: Real-time detection of navigation problems on the World 'Wild' Web. Int. J. Hum. Comput. Stud. 101, 1-9 (2016)

16. W3C: WAI-ARIA Overview (2018). http://www.w3.org/WAI/intro/aria.php

17. WAI: Involving Users in Evaluating Web Accessibility (2010). http://www.w3.org/WAI/ eval/users.html

Open Access This chapter is licensed under the terms of the Creative Commons Attribution 4.0 International License (http://creativecommons.org/licenses/by/4.0/), which permits use, sharing, adaptation, distribution and reproduction in any medium or format, as long as you give appropriate credit to the original author(s) and the source, provide a link to the Creative Commons license and indicate if changes were made.

The images or other third party material in this chapter are included in the chapter's Creative Commons license, unless indicated otherwise in a credit line to the material. If material is not included in the chapter's Creative Commons license and your intended use is not permitted by statutory regulation or exceeds the permitted use, you will need to obtain permission directly from the copyright holder. 